\title{
Component Contribution to the Total Reliability of the WAMS Network
}

\author{
M. I. Uspensky*
}

Institute for Social, Economic and Energy Problems of the North, Federal Research Center of the Komi Science Center of the Ural Branch of the Russian Academy of Sciences, Syktyvkar, Russia.

\begin{abstract}
Nowadays, the control of power systems relies on wide-area monitoring and control system (WAMS), which continuously measures and registers state vector values and is synchronized by signals from the uniform time system. A significant part of this system is the local information network, whose reliability largely determines the proper functioning of WAMS. One can assess the said reliability by dividing it into components. These are hardware or technical reliability associated with failure (destruction) of transmission channel elements or the integrity of communication lines, traffic reliability determined by time loss or data distortion without failure of a transmission channel element, software reliability related to errors in the development of exchange execution programs, and resilience against an external deliberate impact on the transmitted information. This paper addresses the assessment of the first three reliability components of the information network, shows its total value, and estimates the contribution of each component. The last component (resistance to an external deliberate action) is described in a huge number of works, which is why it is not considered in this paper.
\end{abstract}

Index Terms: Wide-area monitoring and control system, local infor-mation network, hardware and software reliability and availability, traffic availability.

\section{INTRODUCTION}

The need for a correct estimation of power system state has led to the creation of a hierarchical system for monitoring transient conditions, i.e., a wide-area monitoring and control system (WAMS). It is based on the technology for measuring phasors (phase vectors) to collect vector information with the aid of phasor measurement

\footnotetext{
${ }^{*}$ Corresponding author.

E-mail: uspensky@energy.komisc.ru
}

http://dx.doi.org/10.38028/esr.2021.01.0004

Received April 13, 2021. Revised April 17, 2021.

Accepted April 25, 2021. Available online May 26, 2021.

This is an open access article under a Creative Commons Attribution-NonCommercial 4.0 International License.

C) 2021 ESI SB RAS and authors. All rights reserved. units (PMUs) using the signals from the global navigation systems that provide simultaneous measurement of phasors [1]. WAMS includes measuring transformers (PMUs), phasor data concentrators (PDCs), and equipment of the local information network (LIN). It allows us to control power system behavior by continuously observing system events. The WAMS reliability is determined by the reliability of every monitoring system component.

The network failure is determined by the loss of terminal communication, which implies not only the absence of such communication but also the distortion of the transmitted information. Then the network reliability includes four components. These are 1) hardware or technical reliability associated with a failure of transmission channel components or destruction of the integrity of communication lines, 2) traffic reliability determined by the time loss or distortion of data without a failure of the transmission channel component, 3) software reliability related to errors in the development of exchange execution programs, and 4) resistance to external actions targeted on the transmitted information.

The paper presents an approach to assessing three first components. An algorithm and implementation of this approach are considered on the example of a 10-node power system. Some features of the information network model are noted.

\section{HARDWARE RELIABILITY OF WAMS NETWORK}

The hardware of WAMS network comprises network connections, electronics of PMUs and PDCs. Since the operation of PDC central processor and communication interface during duplication is similar to the operation of these components in PMU, we will use the reliability assessment of these blocks in [2] obtained from the system of Markov equations of state probabilities, given different lengths of the main and redundant communication channels. Then the availability of communication link of a network consisting of a duplicated information source (PMU, PDC, or, if necessary, an intermediate amplifier) and communication channel lines can be defined as

where

$$
A_{\text {ch }}=A_{P D C} \cdot A_{\text {com }}
$$

$$
A_{P D C}=\frac{\mu_{P D C}^{2}}{\left(\mu_{P D C}+\lambda_{P D C}\right)^{2}},
$$


Table 1. C37.118-2011 frame structure.

\begin{tabular}{lc}
\hline \hline \multicolumn{1}{c}{ Field } & Size \\
\hline Sync byte (SYNC) & 2 bytes \\
\hline Byte number of frame (FRAMESIZE) & 2 bytes \\
\hline PMU Identifier (IDCODE) & 2 bytes \\
\hline Seconds of counting (SOC) & 4 bytes \\
\hline Second fraction/quality flag (FRACSEC) & 4 bytes \\
\hline Status flag (STAT) & 2 bytes \\
\hline Vectors (PHASORS) & $8 \cdot n$ bytes (floating point) \\
\hline Frequency (FREQ) & 4 bytes (floating point) \\
\hline Frequency change rate (DFREQ) & 4 bytes (floating point) \\
\hline Analog data (ANALOG) & $8 \cdot m$ bytes (floating point) \\
\hline Digital data (DIGITAL) & $2 \cdot l$ bytes (discrete values) \\
\hline Cyclic Redundancy Check (CHK) & 2 bytes \\
\hline$l$ is the number of discrete information sources; $m$ is the number of analog information sources; $n$ is synchronized vectors (magnitude and phase). \\
\hline \hline
\end{tabular}

Table 2. Required channel bandwidth, Kbit/s.

\begin{tabular}{ccccc}
\hline \hline \multirow{2}{*}{ Samples per second } & \multicolumn{4}{c}{ Number of PMUs } \\
\cline { 2 - 5 } & 2 & 10 & 40 & 100 \\
\hline 25 & 50 & 249 & 997 & 2392 \\
\hline 50 & 100 & 499 & 1994 & 4984 \\
\hline 100 & 200 & 997 & 3988 & 9969 \\
\hline \hline
\end{tabular}

since PDCs are of the same type, and

$$
A_{\text {com }}=\frac{\mu_{m l} \cdot \mu_{b l}}{\left(\mu_{m l}+\lambda_{m l}\right)\left(\mu_{r l}+\lambda_{r l}\right)} .
$$

Here $A_{P D C}$ is the availability of the redundant information source; $\lambda_{P D C}$ and $\mu_{P D C}$ are failure and recovery rates of the source, respectively. The physical availability of each element of the information carriers (twisted pair, optical fiber, high-frequency channel) is characterized by length $l_{i}$, specific failure rate $\lambda_{m l}$ for the main line and $\lambda_{r l}$ for the redundant line, and the average recovery time $r_{m l}$ for the main and $r_{r l}$ for the redundant line $i$ per unit length. Since reliability indices of communication lines $\lambda_{l}$ and $r_{l}$ approximately linearly depend on their length, and $\mu_{l}=1$ / $r_{l}$, it is easy to evaluate the working state probability of an information carrier element ( $i$-th line availability) as

$$
A_{l n, i}=\frac{\mu_{l n, i}}{\mu_{l n, i}+\lambda_{l n, i}}=\frac{1 /\left(r_{l n, i} \cdot l_{i}\right)}{1 /\left(r_{l n, i} \cdot l_{i}\right)+\lambda_{l n, i} \cdot l_{i}}=\frac{1}{1+\lambda_{l n, i} \cdot r_{l n, i} \cdot l_{i}^{2}} \text {. }
$$

It is worth noting that $r_{l n, i}$ includes two components: the distance-dependent failure search variable, and the recoveryrelated constant. Since the second component has small values, we neglect it. Consequently, the availability of the communication line is inversely proportional to its squared length. In contrast to duplication in electronics, where the backup device usually repeats the basic one, the storage media are most often duplicated by the elements of various reliability indices. This is because under normal conditions, communication is provided via the shortest line in the communication network, and in the case of redundancy, the information goes through the line remaining in the communication network, which can be significantly longer than the main one. Moreover, the approach to solving such a problem is the same as in the case of duplicating electronic units (2) but considers different values of $\lambda_{j}$ and $\mu_{j}$ for the $j$-th communication line (3).

\section{TRAFFIC RELIABILITY}

The traffic reliability lies in timely information transmission, without loss and distortion associated with the exchange channel loading. Traffic-related losses are associated with an unacceptable delay or loss of some information due to the information channel overload but are not associated with the failure of channel device elements, which is taken into account in hardware reliability. Therefore, the traffic reliability is determined by the choice of channel capacity, given a delay in the transmitted information.

The information frame from the generation unit or power line, formed by each PMU, combines 9 vector measurements ( 3 currents and 3 voltages (magnitude and phase), 3 active and reactive power components); 2 analog values (generator current and voltage); the state of PMU, and the state of switching components. The transmission packet also includes the frequency and speed of its change, the time stamp, and the binding for interaction with the information network in the standard C.37.118-2011. The data frame structure is given in Table 1. The amount of information from one PMU takes $b_{i n}=8 \cdot 9+2 \cdot 8+2+2$ $=92$ (bytes). The amount of information per frame of one node (the first six points in Table 1 ) is $b_{f r}=6+8+8+2=24$ (bytes). Depending on the number of PMUs (information sources of measurement), and transmitted measurements per second, the packet volume often varies from 100 to 400 bytes. The approximate channel bandwidth in Kbit/s, depending on the number of source devices and sampling rate, given a margin of 10 percent, is indicated in Table 2 [3]. In this case, $1 \mathrm{Kbit}=1024$ bit.

Information delay is associated with both the type of the exchange channel and the time of unloading its receiving buffer. Packet delivery to a receiver requires time $T_{d}$, which, in the general case, is determined by the signal propagation time $T_{s p}$, the time of packet transmission over the communication line $T_{p t}$ and the packet waiting time $T_{w p}$ in the queue in the communication unit

$$
T_{d}=T_{s p}+T_{p t}+T_{w p}
$$

The signal propagation time, $T_{s p}$ in most communication systems is determined by the propagation time of the electric or optical signal (electromagnetic field). The pulse 


\section{Set power system scheme and initial conditions}

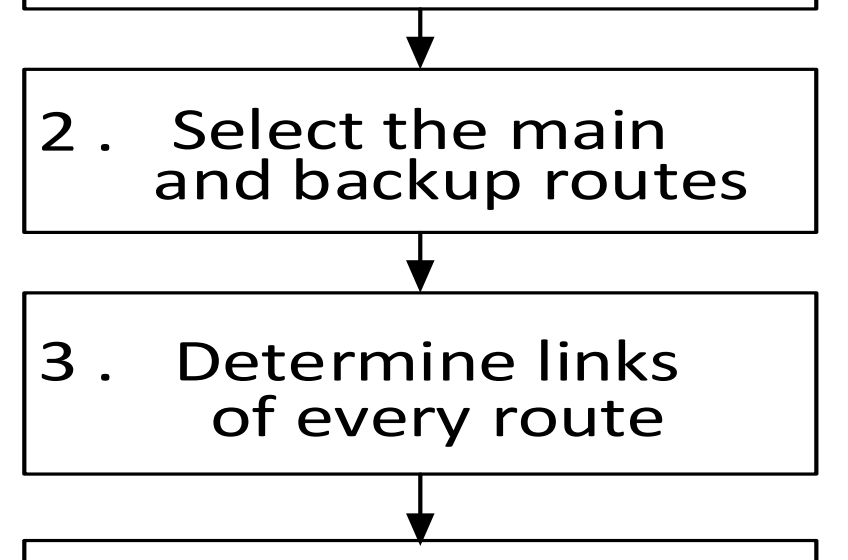

4. Calculate information loads of route links under normal and emergency conditions
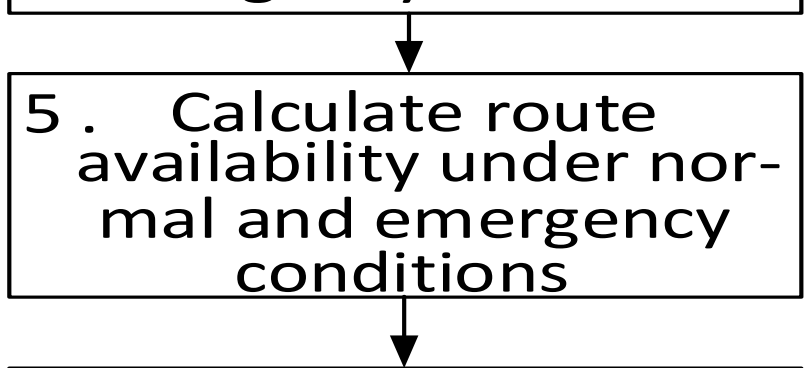

6. Calculate route availability taking into account redundancy

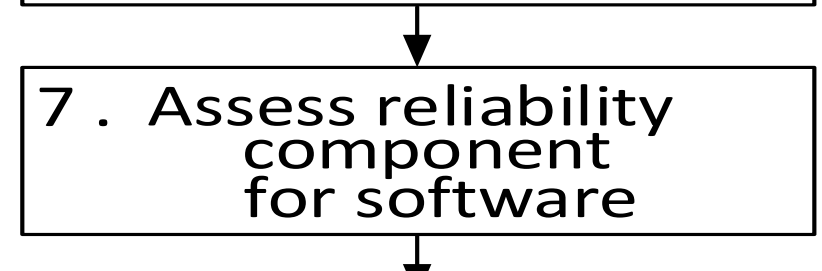

\section{Assess resistance to external Impacts on information}

\section{$\checkmark$}

9. Conclude on acceptability of the option

Fig. 1. Algorithm to research the information network reliability. delay in the optical fiber is $(3.5-5) \cdot l$ (ns) [4] and in the copper wire $5 \cdot l(\mu \mathrm{s})[5]$, where $l$ is the channel length in $\mathrm{km}$.

The packet transmission time, $T_{p t}$, depends on the rate of data transfer via the communication line $v_{t r}(\mathrm{Kbit} / \mathrm{s})$ and the packet volume or length $L_{p}$ (Kbit)

$$
T_{p t}=L_{p} / v_{t r} \text {. }
$$

The propagation speed depends only on the channel material. Therefore, the propagation time along the channel is constant. Transmission time depends only on the packet length.

The main idea behind the design of a data transmission network is to ensure the balance between traffic (the flow of requests $\lambda$, in our case, the measurement frequency), the number of network resources (bandwidth) and the service quality (service flow $\mu$, parameter of request processing). Solving this problem involves considering two levels of the open system interaction model (OSI): network and channel.

Network level. Traffic routes over the network are considered at the network level. To this end, it is convenient to describe the communication network as a graph model [6] (in this case, non-oriented), in which the network nodes (routers) correspond to the graph vertices and the communication lines correspond to the graph arcs. The transmission time to the receiving node is the time spent by the packet in the network line. This time is random to a certain extent.

The intensity of load on the arcs of the network graph $\rho_{i j}$ is determined by the ratio of the intensity of request flow from the information source node $i$ to the intensity of the service flow from the destination node $j\left(\lambda_{i} / \mu_{j}\right)$ and depends on the number of devices and the amount of information from each device. In our case, the request flow intensity is determined by the frequency of parameter measurements at the nodes of the power system $\lambda=f_{m s r}=1 / T_{m s r}$, service flow intensity is determined by the reciprocals of the packet delivery time $\mu=1 / T_{d}=1 /\left(L_{p} / v_{t r}+T_{p c}\right)$, and since this time is shorter than the request period, $T_{w p}=0$. On the other hand, the receiver electronics creates an additional delay, $T_{r e}$, of about $5 \mu$ s on average. Then

$$
\rho_{i j}=\frac{L_{p} / v_{t r}+T_{s p}+T_{r e}}{T_{m s r} .} .
$$

Channel level. This level requires the evaluation of the necessary bandwidth of communication lines between network nodes. In the general case, an approximate formula can be used to estimate the probability of losses [7]

$$
q_{i j}=\frac{1-\rho_{i j}}{1-\rho_{i j}^{N_{j}+1}} \rho_{i j}^{N_{j}},
$$

where $N_{j}$ is the number of sections in the receiver storage $j$; $\rho_{i j}$ is the load intensity of line $i j$.

The absence of loss is determined as

$$
p_{i j}=1-q_{i j} \text {. }
$$

Such an assessment corresponds to one information line connecting two nodes. Taking into account the sequence of 
switching the communication lines of two nodes passing through the intermediate nodes, the overall assessment of the information loss probability is determined as

$$
Q_{\Sigma}=1-\prod_{i j} p_{i j}=1-\prod_{i j}\left(1-q_{i j}\right) .
$$

\section{FAILURE OF THE SOFTWARE}

The failure of the software (SW) is associated with its inconsistency with the objectives set. There are many definitions of software reliability. The most acceptable definition seems to be as follows Software reliability is the probability that the program will work without failures for a certain time, given the degree of their influence on the output results [8].

The frequency of statistical data errors reduced to 100 percent is given in Table 3 with a detailed description of "Incomplete or erroneous task."

The software is not subject to wear and tear and its reliability is determined only by the development errors. Thus, over time, this index should increase if the correction of detected errors does not introduce new errors.

Table 3. Frequency of some types of errors [9].

\begin{tabular}{lc}
\hline \hline \multicolumn{1}{c}{ Cause of error } & Frequency, $\%$ \\
\hline Deviation from the task & 12 \\
\hline Neglect of programming rules & 10 \\
\hline Incorrect data sampling & 10 \\
\hline Erroneous logic or sequence of & 12 \\
operations & 9 \\
\hline Erroneous arithmetic operations & 4 \\
\hline Lack of time to resolve & 4 \\
\hline Incorrect interrupt handling & 3 \\
\hline Invalid constants or source data & 8 \\
\hline Inaccurate recording & 28 \\
\hline Incomplete or erroneous task & $\Downarrow$ \\
\hline Errors in numeric values & 12 \\
\hline Insufficient accuracy requirements & 4 \\
\hline Erroneous characters or signs & 2 \\
\hline Registration errors & 15 \\
\hline Incorrect hardware description & 2 \\
\hline Incomplete or inaccurate development basics & 52 \\
\hline Ambiguity of requirements & 13 \\
\hline \hline
\end{tabular}

Table 4. Routes for the main and redundant information exchange channels.

\begin{tabular}{clc}
\hline \hline Source node & \multicolumn{1}{c}{ Main channel } & Redundant channel \\
\hline 1 & $1-7-4$ & $1-9-8-6-4$ \\
\hline 2 & $2-7-4$ & $2-9-7-4$ \\
\hline 3 & $3-4$ & $3-5-4$ \\
\hline 5 & $5-4$ & $5-6-4$ \\
\hline 6 & $6-4$ & $6-5-4$ \\
\hline 7 & $7-4$ & $7-6-4$ \\
\hline 8 & $8-6-4$ & $8-9-7-4$ \\
\hline 9 & $9-7-4$ & $9-8-6-4$ \\
\hline 10 & $10-2-7-4$ & - \\
\hline \hline
\end{tabular}

For critical applications, which should include the WAMS software, by the time the system is delivered to the client, it may contain from 4 to 15 errors per 100000 lines of program code [10]. For clarity, we note that the number of code lines of WINDOWS XP is above 45 million, NASA has 40 million code lines, and Linux 4.11 kernel has more than 18 million code lines [11].

When evaluating the WAMS program of 10 million lines of code, the number of errors at the beginning of program operation is $E=(V / 100000) \cdot 4=400$ (errors). Then, using the formula for the mean time between the software failures, we get

or

$$
\lambda_{S W}=\beta \frac{E}{V}=0.01 \frac{400}{10^{7}}=4 \cdot 10^{-7}
$$

$$
t_{S W}=\frac{1}{\lambda_{S W} \cdot 8760}=\frac{10^{7}}{4 \cdot 8760} \approx 285,
$$

where $E$ is the number of errors per program accepted for operation, $V$ is the program volume in lines of code, $\beta$ is the program complexity coefficient, usually in the range of 0.001 to $0.01, \lambda_{S W}$ is the failure rate and $t_{S W}$ is the mean time between the software failures (years), 8760 is the number of hours per year. With a value of one error per 1000 code lines, accepted for applied software after testing with the same number of code lines, $E=10000$ errors:

$$
\lambda_{S W}=\beta \frac{E}{V}=0.01 \frac{10000}{10^{7}}=10^{-5}
$$

or

$$
t_{S W}=\frac{1}{\lambda_{S W} \cdot 8760}=\frac{10^{5}}{8760} \approx 11.4
$$

or about one failure in 12 years.

\section{PROCEDURE FOR ASSESSING THE LIN RELIABILITY}

Since the reliability of the local information network (LIN) is investigated for a given scheme, the research algorithm is as follows (Fig. 1).

1. Set a scheme of information exchange in the form of links between nodes, the length of links, and the type of links (wired, fiber-optic, high-frequency, etc.). Set also the initial conditions. These are the failure and recovery rates of information sources (PMUs) $\lambda_{i s}$ and $\mu_{i s}$, respectively; specific failure rates of the main and redundant lines $\left(\lambda_{m l}\right.$ and $\left.\lambda_{r l}\right)$; the average recovery time of the main and redundant lines $\left(r_{m l}\right.$ and $\left.r_{r l}\right)$ per unit of length; propagation delay $T_{s p}$; delay in electronic devices $T_{r e}$; transmission rate $v_{t r}$; transmission frequency or measurement period $T_{m s r}$, and the number of information sources at each node. A link means a connection between the adjacent nodes in the network.

2. Select the main and backup routes for the information exchange between the sources and the dispatching point. The main route is usually determined by the shortest path from the information source to the dispatching point, the backup one depends on the failed communication link. 
3. Determine a route by its set of links.

4. Calculate information loads of links of every route under normal and emergency conditions in terms of link failure.

5. Based on the prepared information, calculate the hardware availability and traffic reliability of the routes under normal and emergency conditions.

6. Calculate the availability of routes taking into account redundancy.
7. Estimate the software component, in terms of software development reliability.

8. Assess the resistance to external impact on the information. Mentioning this point, we do not dwell in detail on such an assessment but refer the reader to the literary sources, for example, $[12,13]$.

9. Make a conclusion on the acceptability of the option under study and, if necessary, its weak points.

Table 5. Availability of links of fiber-optic information exchange channel.

\begin{tabular}{|c|c|c|c|c|c|c|c|c|c|}
\hline Link & $l, \mathrm{~km}$ & $\lambda_{\text {con }}$, fail./yr. & $r_{\text {con }}, \mathrm{hr} . / \mathrm{recov}$. & $A_{\text {link }}$ & Link & $l, \mathrm{~km}$ & $\lambda_{\text {con }}$, fail./yr. & $r_{\text {con }}, \mathrm{hr} . / \mathrm{recov}$. & $A_{\text {link }}$ \\
\hline $1-7$ & 150.0 & 2.628 & 31.32 & 0.990433883 & $4-6$ & 30.0 & 0.5256 & 6.264 & 0.999364399 \\
\hline $1-9$ & 75.0 & 1.314 & 15.66 & 0.997397114 & $4-7$ & 50.0 & 0.876 & 10.44 & 0.99869736 \\
\hline $2-7$ & 150.0 & 2.628 & 31.32 & 0.990433883 & $5-6$ & 50.0 & 0.876 & 10.44 & 0.99869736 \\
\hline $2-9$ & 75.0 & 1.314 & 15.66 & 0.997397114 & $6-7$ & 47.0 & 0.82344 & 9.8136 & 0.998818611 \\
\hline $2-10$ & 70.0 & 1.2264 & 14.616 & 0.997698469 & $6-8$ & 145.0 & 2.5404 & 30.276 & 0.991038641 \\
\hline $3-4$ & 70.0 & 1.2264 & 14.616 & 0.997698469 & $7-9$ & 130.0 & 2.2776 & 27.144 & 0.99273384 \\
\hline $3-5$ & 50.0 & 0.876 & 10.44 & 0.99869736 & $8-9$ & 40.0 & 0.7008 & 8.352 & 0.99907246 \\
\hline $4-5$ & 40.0 & 0.7008 & 8.352 & 0.99907246 & & & & & \\
\hline
\end{tabular}

Table 6. Availability of the fiber-optic information exchange channel.

\begin{tabular}{clllclll}
\hline \hline Node & $A_{\text {main channel }}$ & A redundant channel & $A_{\text {channel with redundancy }}$ & Node & $A_{\text {main channel }}$ & $A_{\text {redundant channel }}$ & $A_{\text {channel with redundancy }}$ \\
\hline 1 & 0.989400945 & 0.987684744 & 0.99986947 & 7 & 0.990433883 & 0.998443352 & 0.999985109 \\
\hline 2 & 0.989400945 & 0.989374459 & 0.999887379 & 8 & 0.990666305 & 0.991036328 & 0.999916336 \\
\hline 3 & 0.997698469 & 0.998030512 & 0.999995467 & 9 & 0.991698503 & 0.99000482 & 0.999917025 \\
\hline 6 & 0.99907246 & 0.998322147 & 0.999998444 & 10 & 0.987380523 & 0 & 0.987380523 \\
\hline \hline
\end{tabular}

Table 7. Availability of links of the power line information exchange channel.

\begin{tabular}{|c|c|c|c|c|c|c|c|c|c|}
\hline Link & $l, \mathrm{~km}$ & $\lambda_{\text {con }}$, fail./yr. & $r_{c o n}, \mathrm{hr} . / \mathrm{recov}$. & $A_{\text {link }}$ & Link & $l, \mathrm{~km}$ & $\lambda_{\text {con }}$, fail./yr. & $\begin{array}{c}r_{\text {con }}, \\
\mathrm{hr} . / \mathrm{recov} .\end{array}$ & $A_{\text {link }}$ \\
\hline $1-7$ & 150.0 & 2.94 & 28.5 & 0.990268019 & $4-6$ & 30.0 & 0.588 & 5.7 & 0.999357643 \\
\hline $1-9$ & 75.0 & 1.47 & 14.25 & 0.997355058 & $4-7$ & 50.0 & 0.98 & 9.5 & 0.998678619 \\
\hline $2-7$ & 150.0 & 2.94 & 28.5 & 0.990268019 & $5-6$ & 50.0 & 0.98 & 9.5 & 0.998678619 \\
\hline $2-9$ & 75.0 & 1.47 & 14.25 & 0.997355058 & $6-7$ & 47.0 & 0.9212 & 8.93 & 0.998802048 \\
\hline $2-10$ & 70.0 & 1.372 & 13.3 & 0.997661811 & $6-8$ & 145.0 & 2.842 & 27.55 & 0.990883459 \\
\hline $3-4$ & 70.0 & 1.372 & 13.3 & 0.997661811 & $7-9$ & 130.0 & 2.548 & 24.7 & 0.992608673 \\
\hline $3-5$ & 50.0 & 0.98 & 9.5 & 0.998678619 & $8-9$ & 40.0 & 0.784 & 7.6 & 0.999060456 \\
\hline $4-5$ & 40.0 & 0.784 & 7.6 & 0.999060456 & & & & & \\
\hline
\end{tabular}

Table 8. Availability of the power line information exchange channel.

\begin{tabular}{ccccccc}
\hline \hline Node & $A_{\text {main channel }}$ & $A_{\text {redundant channel }}$ & $\begin{array}{c}A_{\text {channel with }} \\
\text { redundancy }\end{array}$ & Node & $A_{\text {main channel }}$ & $A_{\text {redundant channel }}$ \\
\hline 1 & 0.98921669 & 0.987469907 & 0.999864884 & 7 & 0.99026802 & 0.998420046 \\
\hline 2 & 0.98921669 & 0.989189439 & 0.999883426 & 8 & 0.99050449 & 0.990880875 \\
\hline 3 & 0.99766181 & 0.997999793 & 0.999995323 & 9 & 0.99155486 & 0.989831215 \\
\hline 5 & 0.99906046 & 0.998296664 & 0.9999984 & 10 & 0.98716037 & 0.999913409 \\
\hline 6 & 0.99935764 & 0.997999793 & 0.999998715 & & & 0.999914123 \\
\hline \hline
\end{tabular}

Table 9. Resulting $\lambda \Sigma$ and $\mu \Sigma$ values of communication channels.

\begin{tabular}{|c|c|c|c|c|}
\hline \multirow[t]{2}{*}{ Source - node } & \multicolumn{2}{|c|}{ Fiber-optic channels } & \multicolumn{2}{|c|}{ Power line channels } \\
\hline & $\lambda_{\Sigma}$ & $\mu \Sigma$ & $\lambda_{\Sigma}$ & $\mu \Sigma$ \\
\hline 1 & 0.09589628 & 734.572164 & 0.109127394 & 807.5486398 \\
\hline 2 & 0.083695895 & 743.08248 & 0.095243883 & 816.9324165 \\
\hline 3 & 0.006031754 & 1330.67373 & 0.006854215 & 1465.551612 \\
\hline 5 & 0.002472612 & 1588.79738 & 0.00280486 & 1752.642178 \\
\hline 6 & 0.00203475 & 1625.44545 & 0.002306221 & 1794.935514 \\
\hline 7 & 0.016936974 & 1137.37422 & 0.019264457 & 1252.864972 \\
\hline 8 & 0.062884682 & 751.569245 & 0.071559069 & 826.33357 \\
\hline 9 & 0.062221822 & 749.824533 & 0.070804518 & 824.418488 \\
\hline 10 & 0.077536602 & 642.210362 & 0.088232547 & 706.0262268 \\
\hline
\end{tabular}


Table 10. Input data on the information network.

\begin{tabular}{|c|c|c|c|c|c|c|c|}
\hline Link & $l, \mathrm{~km}$ & $b_{\text {in }}$ & $b_{\mathrm{fr}}$ & $\sum b_{i n}^{n r}$ & $\sum b_{f r}^{n r}$ & $\sum b_{i n}^{e m}$ & $\sum b_{f r}^{e m}$ \\
\hline $1-7$ & 150 & 2 & 1 & 2 & 1 & 5 & 3 \\
\hline $2-9$ & 75 & 2 & 1 & 3 & 2 & 5 & 3 \\
\hline $10-2$ & 70 & 1 & 1 & 1 & 1 & 1 & 1 \\
\hline $3-4$ & 70 & 6 & 2 & 6 & 2 & 6 & 2 \\
\hline $3-5$ & 50 & 0 & 0 & 0 & 0 & 6 & 2 \\
\hline $9-7$ & 130 & 1 & 1 & 4 & 3 & 6 & 4 \\
\hline $9-8$ & 40 & 0 & 0 & 0 & 0 & 6 & 4 \\
\hline 8-6 & 145 & 1 & 1 & 1 & 1 & 7 & 5 \\
\hline $7-4$ & 50 & 1 & 1 & 7 & 5 & 7 & 5 \\
\hline 6-5 & 50 & 1 & 1 & 0 & 0 & 7 & 3 \\
\hline 6-4 & 30 & 6 & 2 & 7 & 3 & 13 & 7 \\
\hline $5-4$ & 40 & 1 & 1 & 1 & 1 & 8 & 4 \\
\hline $7-6$ & 50 & 6 & 2 & 0 & 0 & 7 & 5 \\
\hline $2-7$ & 150 & 0 & 0 & 0 & 0 & 5 & 3 \\
\hline $1-9$ & 75 & 0 & 0 & 0 & 0 & 5 & 3 \\
\hline
\end{tabular}

Table 11. Loads $\rho_{i j}$ and probabilities $q_{i j}$ of information loss of an individual link.

\begin{tabular}{ccccc}
\hline \hline Link & $\rho_{i j}^{n r}$ & $q_{i j}^{n r}$ & $\rho_{i j}^{e m}$ & $q_{i j}^{e m}$ \\
\hline $1-7$ & 0.01593 & $1.008 \mathrm{E}-09$ & 0.04065 & $1.0643 \mathrm{E}-07$ \\
\hline $2-9$ & 0.02477 & $9.099 \mathrm{E}-09$ & 0.04064 & $1.0638 \mathrm{E}-07$ \\
\hline $10-2$ & 0.00890 & $5.545 \mathrm{E}-11$ & 0.00890 & $5.5455 \mathrm{E}-11$ \\
\hline $3-4$ & 0.04583 & $1.929 \mathrm{E}-07$ & 0.04580 & $1.9296 \mathrm{E}-07$ \\
\hline $3-5$ & - & - & 0.04583 & $1.9289 \mathrm{E}-07$ \\
\hline $9-7$ & 0.03363 & $4.154 \mathrm{E}-08$ & 0.04949 & $2.8233 \mathrm{E}-07$ \\
\hline $9-8$ & - & - & 0,04949 & $2.8221 \mathrm{E}-07$ \\
\hline $8-6$ & 0.00891 & $5.557 \mathrm{E}-11$ & 0.05835 & $6.3671 \mathrm{E}-07$ \\
\hline $7-4$ & 0.05834 & $6.364 \mathrm{E}-07$ & 0.05834 & $4.6204 \mathrm{E}-07$ \\
\hline $6-5$ & - & - & 0.05468 & $1.0961 \mathrm{E}-05$ \\
\hline $6-4$ & 0.05468 & $4.619 \mathrm{E}-07$ & 0.10412 & $9.6904 \mathrm{E}-07$ \\
\hline $5-4$ & 0.00890 & $5.541 \mathrm{E}-11$ & 0.06353 & $6.3644 \mathrm{E}-07$ \\
\hline $7-6$ & - & - & 0.05834 & $1.0649 \mathrm{E}-07$ \\
\hline $2-7$ & - & - & 0.04065 & $1.0638 \mathrm{E}-07$ \\
\hline $1-9$ & - & - & 0.04064 & \\
\hline \hline
\end{tabular}

\section{POWER SYSTEM MODEL WITH WAMS}

Let us consider the described approach on the example of a 10-node system considered in [14], Fig. 2. Without dwelling on the optimal composition of PMUs, we will assign PMU to each node of the network and select sites for PDCs at nodes 4 and 9 . We determine the main and redundant channels of information exchange from the PMU of each node (Table 4 and Fig. 3). Fig. 4a indicates such links without redundancy, and Fig. 4b presents them with redundancy. For fiber-optic communication lines, the specific indices according to Table 12.4 from [15] and data from $[16,17]$ are $\lambda_{l}=0.01752$ failure/(km/yr.); $r_{l}=0.2088$ $\mathrm{hr} . /(\mathrm{km} /$ recovery). Reliability indices of electronic devices with their duplication are $\lambda_{P M U}=1.539 \cdot 10^{-3}$ failure/yr., $\mu_{P M U}=5.922$ recovery/yr., $A_{P M U}=0.999740$ [2], $\lambda_{P D C}=$ $2.673 \cdot 10^{-6}$ failure/yr. and $\mu_{P D C}=740$ recovery/yr., $A_{P D C}=$ 0.999999996 [15].

\section{TECHNICAL AVAILABILITY OF LOCAL INFORMATION NETWORK}

Table 5 presents the determined link availabilities of the information exchange channel, each including an information source (PMU or PDC) and the actual fiber-optic connection, given that $\mu_{\text {con }}=8760 / r_{\text {con }}$ recovery/ yr. Then the availability of individual information channel is determined as the product of availabilities of sequential links, which corresponds to a channel without redundancy, and the availability of the $i$-th channel with redundancy is calculated as

$$
A_{c h, i}=1-\left(1-A_{m_{-} c h, i}\right) \cdot\left(1-A_{r_{-} c h, i}\right),
$$

where $A_{m_{c} c h, i}$ is the $i$-th main channel connection availability, and $A_{r \text { ch,i }}$ is the $i$-th redundant channel connection availability. The channel availabilities are given in Table 6 according to the connections (Table 4) and to the availabilities of the links (Table 5).

Let the line from the source to the dispatching node be a "channel" consisting of links between neighboring nodes. Table 6 shows that with a single set of links of communication channel, its availability varies in the range of one to three nines after the decimal point. The availability of communication with redundancy is maintained at the level of three nines after the decimal point, even if a source is located sufficiently far from the dispatching node, such as nodes 1 and 2 . The availability of channel 10 is the value of the main channel availability since this channel has no redundancy, i.e., redundant communication lines. 
Table 12. Probabilities of the route information loss.

\begin{tabular}{cccc}
\hline \hline Route & $Q_{\Sigma_{u}}^{n r}$ & Route & $Q_{\Sigma_{u}}^{\text {em }}$ \\
\hline $1-7-4$ & $6.37 \mathrm{E}-07$ & $1-9-8-6-4$ & $1.199 \mathrm{E}-05$ \\
\hline $2-9-7-4$ & $6.87 \mathrm{E}-07$ & $2-7-4$ & $7.429 \mathrm{E}-07$ \\
\hline $3-4$ & $1.93 \mathrm{E}-07$ & $3-5-4$ & $1.162 \mathrm{E}-06$ \\
\hline $5-4$ & $5.54 \mathrm{E}-11$ & $5-6-4$ & $1.142 \mathrm{E}-05$ \\
\hline $6-4$ & $4.62 \mathrm{E}-07$ & $6-5-4$ & $1.431 \mathrm{E}-06$ \\
\hline $7-4$ & $6.36 \mathrm{E}-07$ & $8-6-4$ & $1.16 \mathrm{E}-05$ \\
\hline $8-6-4$ & $4.62 \mathrm{E}-07$ & $9-8-6-4$ & $1.201 \mathrm{E}-06$ \\
\hline $9-7-4$ & $6.78 \mathrm{E}-07$ & - & $1.188 \mathrm{E}-05$ \\
\hline $10-2-7-4$ & $6.37 \mathrm{E}-07$ & - \\
\hline
\end{tabular}

Table 13. Influence of load intensity $\rho$ and the number of sections $N$

on the probability of information loss $q$ and error-free operation $p$ for link 7-4.

\begin{tabular}{|c|c|c|c|c|c|c|c|c|c|}
\hline \# & 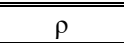 & $\bar{N} N$ & $\overline{p p}$ & $\bar{q}$ & $\overline{~ \# ~}$ & 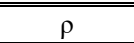 & $\bar{N}$ & $\overline{p p}$ & $\bar{q}$ \\
\hline 1 & \multirow{7}{*}{0.01} & 0 & 0 & 1 & 5 & \multirow{3}{*}{0.3} & 7 & 0.9998469 & 0.0001531 \\
\hline 2 & & 1 & 0.99009901 & 0.00990099 & 6 & & 10 & 0.999995867 & $4.13344 \mathrm{E}-06$ \\
\hline 3 & & 3 & 0.99999901 & $9.9 \mathrm{E}-07$ & 7 & & 100 & 1 & 0 \\
\hline 4 & & 5 & 1 & $9.9 \mathrm{E}-11$ & 1 & \multirow{7}{*}{0.5} & 0 & 0 & 1 \\
\hline 5 & & 7 & 1 & $9.88098 \mathrm{E}-15$ & 2 & & 1 & 0.666666667 & 0.333333333 \\
\hline 6 & & 10 & 1 & 0 & 3 & & 3 & 0.933333333 & 0.066666667 \\
\hline 7 & & 100 & 1 & 0 & 4 & & 5 & 0.984126984 & 0.015873016 \\
\hline 1 & \multirow{7}{*}{0.058341} & 0 & 0 & 1 & 5 & & 7 & 0.996078431 & 0.003921569 \\
\hline 2 & & 1 & 0.944874979 & 0.055125021 & 6 & & 10 & 0.99951148 & 0.00048852 \\
\hline 3 & & 3 & 0.999813008 & 0.000186992 & 7 & & 100 & 1 & 0 \\
\hline 4 & & 5 & 0.999999364 & $6.36452 \mathrm{E}-07$ & 1 & \multirow{7}{*}{0.7} & 0 & 0 & 1 \\
\hline 5 & & 7 & 0.999999998 & $2.16628 \mathrm{E}-09$ & 2 & & 1 & 0.588235294 & 0.411764706 \\
\hline 6 & & 10 & 1 & $4.30211 \mathrm{E}-13$ & 3 & & 3 & 0.864587446 & 0.135412554 \\
\hline 7 & & 100 & 1 & 0 & 4 & & 5 & 0.942856074 & 0.057143926 \\
\hline 1 & \multirow{7}{*}{0.1} & 0 & 0.000000000 & 1.000000000 & 5 & & 7 & 0.973782312 & 0.026217688 \\
\hline 2 & & 1 & 0.909090909 & 0.090909090 & 6 & & 10 & 0.991354799 & 0.008645201 \\
\hline 3 & & 3 & 0.999099909 & 0.000900090 & 7 & & 100 & 1 & $1.11022 \mathrm{E}-16$ \\
\hline 4 & & 5 & 0.999990999 & 0.000009000 & 1 & \multirow{7}{*}{0.9999999} & 0 & 0 & 1 \\
\hline 5 & & 7 & 0.999999909 & 0.000000090 & 2 & & 1 & 0.500000025 & 0.499999975 \\
\hline 6 & & 10 & 0.999999999 & $9.000007 \mathrm{E}-11$ & 3 & & 3 & 0.750000038 & 0.249999962 \\
\hline 7 & & 100 & 1.000000000 & 0.000000000 & 4 & & 5 & 0.833333375 & 0.166666625 \\
\hline 1 & \multirow{4}{*}{0.3} & 0 & 0 & 1 & 5 & & 7 & 0.875000044 & 0.124999956 \\
\hline 2 & & 1 & 0.769230769 & 0.230769231 & 6 & & 10 & 0.909090955 & 0.090909045 \\
\hline 3 & & 3 & 0.98094566 & 0.01905434 & 7 & & 100 & 0.990099059 & 0.009900941 \\
\hline 4 & & 5 & 0.998297759 & 0.001702241 & & & & & \\
\hline
\end{tabular}

The specific indices for communication lines using a high-frequency signal on power lines (Table 12.3 [4]) are $\lambda_{l}$ $=0.0196$ failure $/(\mathrm{km} / \mathrm{yr}.) ; r_{l}=0.19 \mathrm{hr} . /(\mathrm{km} /$ recovery $)$. The rest of the data are the same. The availabilities of channel link and information exchange channels are given in Tables 7 and Table 8, respectively.

A comparison of Tables 5, 6 and 7, and 8 shows that the difference in availability between fiber-optic and highfrequency transmission is negligible. Table 8 shows that for optical fiber only the main channel determines the availability of node 10 .

Based on the sequential connection of links of the main or redundant information channel and parallel operation of these channels on the server, we determine the failure rate $\lambda_{\Sigma}$ and the recovery rate $\mu_{\Sigma}$ for fiber-optic information exchange channels. Then, $\lambda_{i, \Sigma}=\sum_{j} \lambda_{i, j}$, where $i$ is the main or redundant information channel, $j$ is the link of this channel. Further, we determine $\mu_{i, \Sigma}=\frac{\lambda_{i, \Sigma} \cdot A_{c h, i}}{1-A_{c h, i}}$ from the relation $A=\frac{\mu}{\mu+\lambda}$ and find $\mu_{\Sigma}=\sum_{i} \mu_{i, \Sigma}$, and $\lambda_{\Sigma}=\frac{\mu_{\Sigma}\left(1-A_{c h, i}\right)}{A_{c h, i}}$. The resulting $\lambda_{\Sigma}$ and $\mu_{\Sigma}$ for fiber-optic and power lines are summarized in Table 9.

With a complex network of information connections, we can find a redundant connection from the server node to the node with failed connection, excluding the latter one. To do this, we use the depth-first and breadth-first search algorithm as proposed in [17]. It allows finding a backup path with failed connections, if one exists, or warning about its absence. When searching, the column "Redundant channel" is built in Table 4, and then the hardware reliability is evaluated for the found path. These backup routes are stored in Table 1 in the order of 


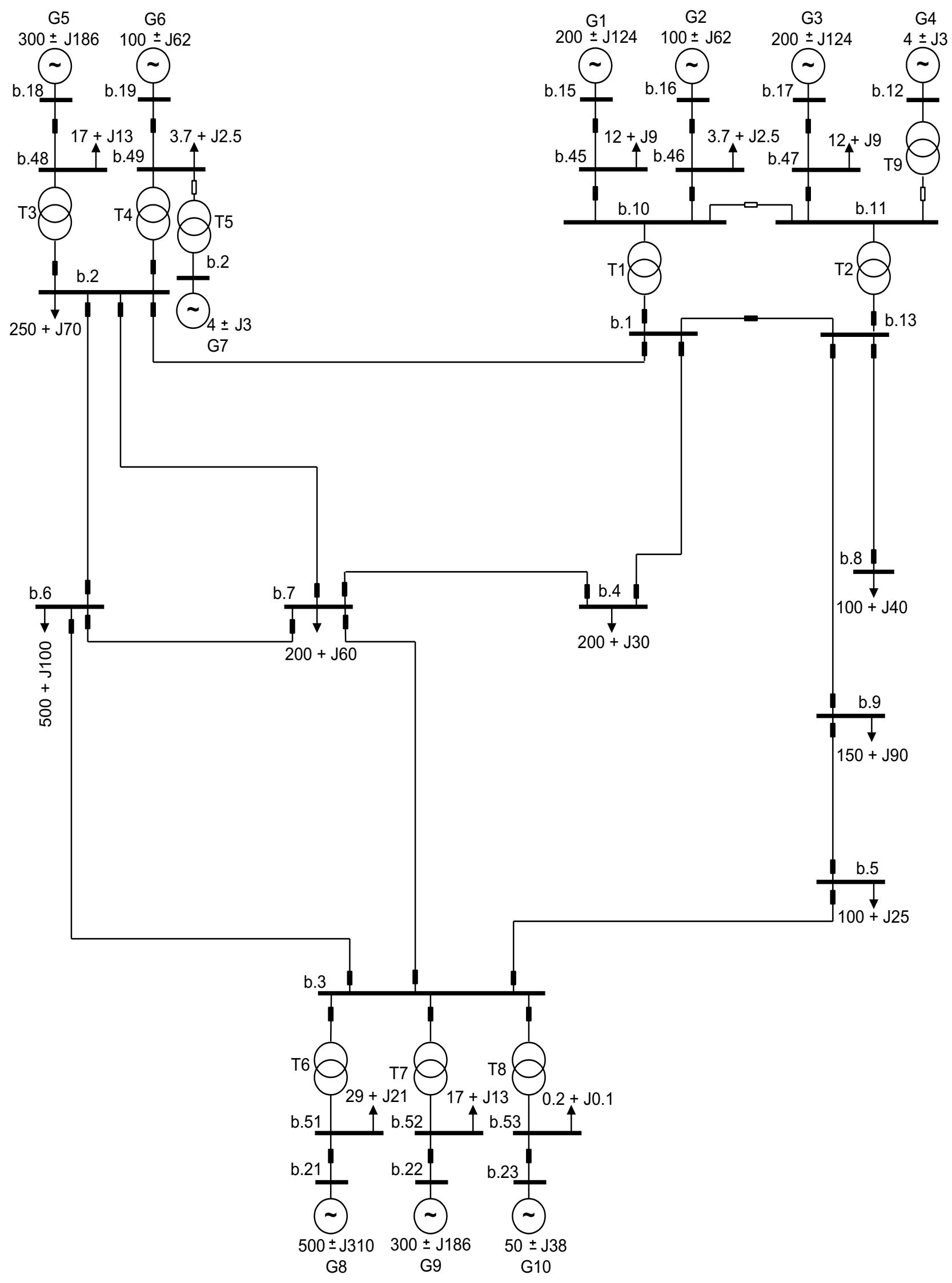

Fig. 2. Model diagram of the test power system. The black switch is on, the white one is off. 


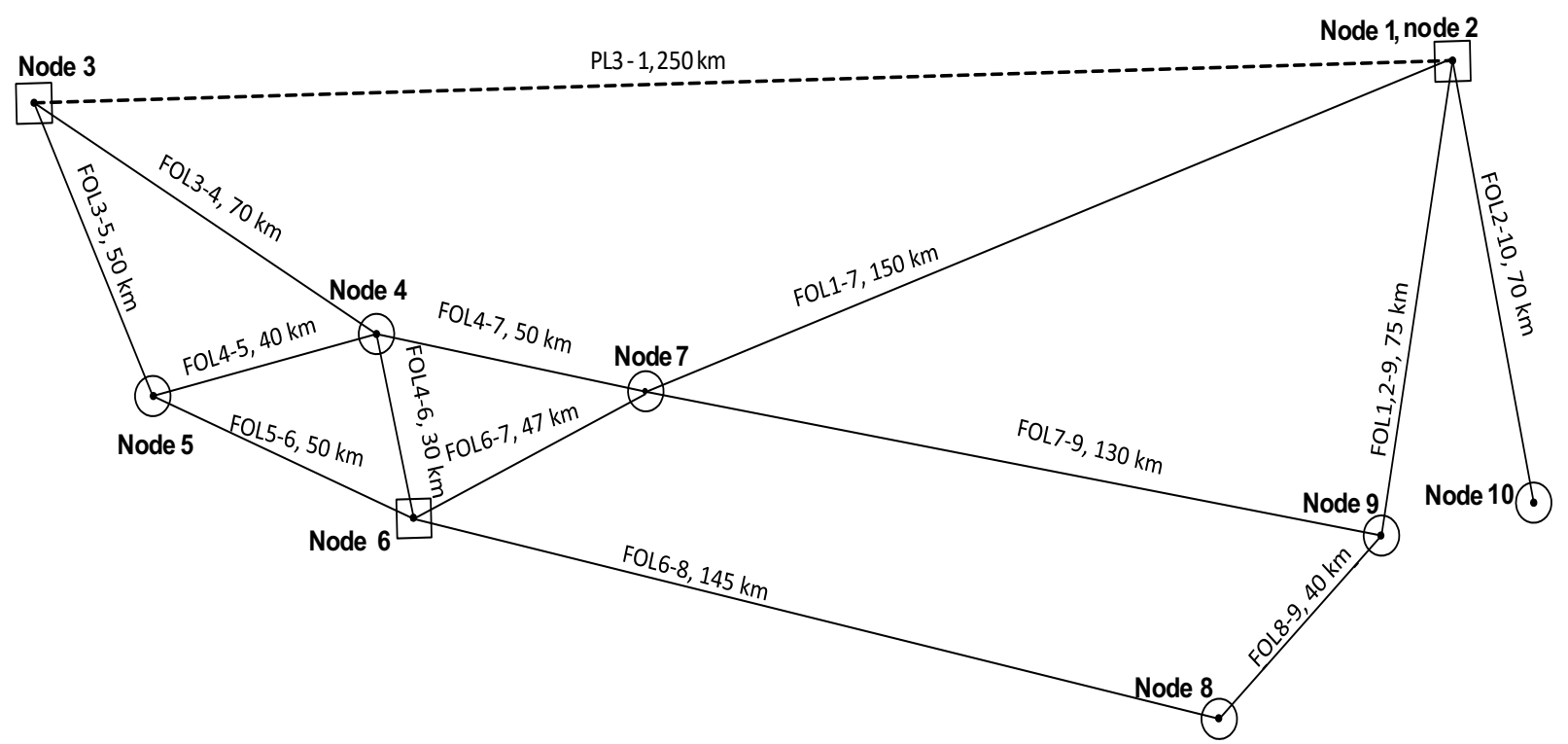

Fig. 3. The geographical location of the test power system (scale: $1 \mathrm{~cm}=20 \mathrm{~km}$ ). Rectangle nodes contain generation. Circular nodes have only load.

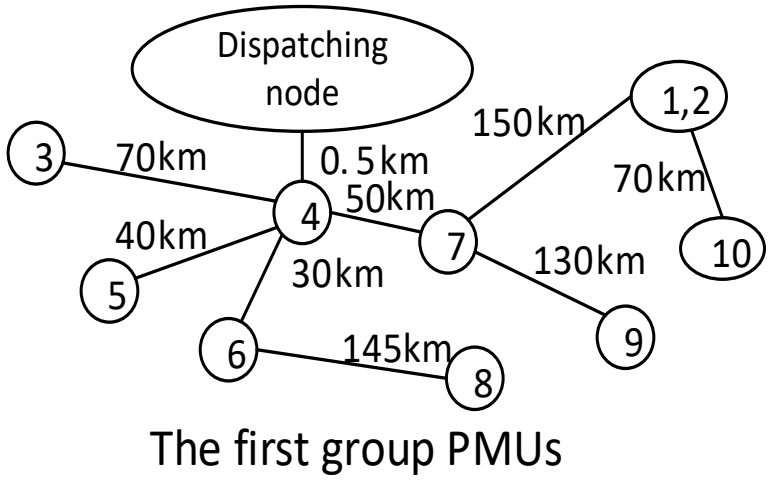

a)

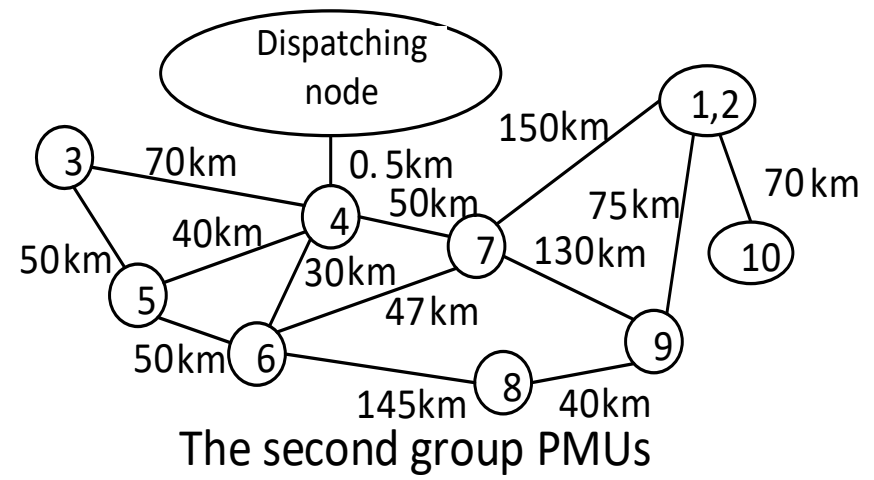

b)

Fig. 4. Communication channels: a) without redundancy, b) with redundancy.

Table 14. An example of the difference between the values of two schemes by contribution of reliability components.

\begin{tabular}{ccccccc}
\hline$R_{1}$ & $R_{2}$ & $R_{3}$ & $R_{4}$ & $Q_{a}$ & $Q_{b}$ & $\frac{Q_{b}-Q_{a}}{Q_{a}} 100 \%$ \\
\hline 0.9 & 0.9 & 0.9 & 0.9 & 0.0199 & 0.0361 & 44.87534626 \\
\hline 0.9 & 0.8 & 0.8 & 0.9 & 0.0396 & 0.0784 & 49.48979592 \\
\hline 0.8 & 0.9 & 0.8 & 0.9 & 0.0396 & 0.0684 & 42.10526316 \\
\hline 0.8 & 0.8 & 0.8 & 0.8 & 0.0784 & 0.1296 & 39.50617284 \\
\hline \hline
\end{tabular}

Table 15. The difference in the reliability component contributions for two equivalent circuits.

\begin{tabular}{ccccccc}
\hline \hline \multirow{2}{*}{$A_{\text {main }}$} & $A_{\text {redundant }}$ & $P_{n r}$ & $P_{\text {em }}$ & $Q_{\text {paral. }}$ & $Q_{\text {sequen. }}$ & $\frac{Q_{\text {sequen. }}-Q_{\text {paral. }}}{100 \%}$ \\
& & & & & & $Q_{\text {paral. }}$ \\
\hline 0.989400945 & 0.987684744 & 0.999999363 & 0.99998801 & 0.00013053 & 0.000130663 & 0.102001899 \\
\hline 0.989400945 & 0.989374459 & 0.999999313 & 0.9999992571 & 0.000112621 & 0.000112636 & 0.013328578 \\
\hline 0.997698469 & 0.998030512 & 0.999999807 & 0.999998838 & $4.53284 \mathrm{E}-06$ & $4.53589 \mathrm{E}-06$ & 0.067205108 \\
\hline 0.99907246 & 0.998322147 & 0.9999999999 & 0.99998569 & $1.55628 \mathrm{E}-06$ & $1.56953 \mathrm{E}-06$ & 0.844266885 \\
\hline 0.999364399 & 0.998030512 & 0.999999538 & 0.999998569 & $1.25181 \mathrm{E}-06$ & $1.25363 \mathrm{E}-06$ & 0.144945673 \\
\hline 0.990433883 & 0.998443352 & 0.999999364 & 0.9999884 & $1.48911 \mathrm{E}-05$ & $1.50029 \mathrm{E}-05$ & 0.745022642 \\
\hline 0.990666305 & 0.991036328 & 0.999999538 & 0.999998799 & $8.36642 \mathrm{E}-05$ & $8.36794 \mathrm{E}-05$ & 0.018178718 \\
\hline 0.991698503 & 0.99000482 & 0.999999322 & 0.99998812 & $8.2975 \mathrm{E}-05$ & $8.30793 \mathrm{E}-05$ & 0.125610524 \\
\hline 0.987380523 & 0 & 0.999999363 & 0 & 0.012620106 & 0.012620106 & 0 \\
\hline \hline
\end{tabular}


Table 16. Data for calculating the contribution of components.

\begin{tabular}{|c|c|c|c|}
\hline Source node & $\begin{array}{l}A_{\text {channel with }} \\
\text { redundancy }\end{array}$ & $Q_{\Sigma m, i}^{e m}$ & $\frac{1}{t_{S W} \cdot n}$ \\
\hline 1 & 0.99986947 & $1.199 \mathrm{E}-05$ & \multirow{9}{*}{$\begin{array}{c}1 / 285 / 10= \\
3.5087719 \mathrm{E}-04\end{array}$} \\
\hline 2 & 0.999887379 & $7.429 \mathrm{E}-07$ & \\
\hline 3 & 0.999995467 & $1.162 \mathrm{E}-06$ & \\
\hline 5 & 0.999998444 & $1.142 \mathrm{E}-05$ & \\
\hline 6 & 0.999998748 & $1.431 \mathrm{E}-06$ & \\
\hline 7 & 0.990433883 & $1.16 \mathrm{E}-05$ & \\
\hline 8 & 0.990666305 & $1.201 \mathrm{E}-06$ & \\
\hline 9 & 0.991698503 & $1.188 \mathrm{E}-05$ & \\
\hline 10 & 0.987380523 & $6.37 \mathrm{E}-07$ & \\
\hline
\end{tabular}

Table 17. Calculation results of the contribution by component.

\begin{tabular}{|c|c|c|c|c|c|}
\hline Source -node & $\operatorname{Con}_{A, i}, 100 \%$ & $\operatorname{Con}_{Q, i}, 100 \%$ & Con $_{S W, i}, 100 \%$ & $\operatorname{Con}_{\Sigma, i}, 100 \%$ & $A_{\Sigma, i}$ \\
\hline 1 & 73.49342727 & 6.750832704 & 19.75574003 & 0.01776077 & 0.999822399 \\
\hline 2 & 75.86377335 & 0.500432399 & 23.63579425 & 0.01484516 & 0.999851552 \\
\hline 3 & 11.11500184 & 2.849246004 & 86.03575216 & 0.00407827 & 0.999959217 \\
\hline 5 & 3.237369106 & 23.76012545 & 73.00250545 & 0.00480637 & 0.999951937 \\
\hline 6 & 3.31473698 & 3.788649056 & 92.89661396 & 0.00377707 & 0.999962229 \\
\hline 7 & 24.18205538 & 18.83767661 & 56.98026802 & 0.00615787 & 0.999938422 \\
\hline 8 & 69.74748092 & 1.001227823 & 29.25129126 & 0.01199527 & 0.99988005 \\
\hline 9 & 63.85505894 & 9.14248991 & 27.00245115 & 0.01299427 & 0.999870062 \\
\hline 10 & 99.71770723 & 0.005033503 & 0.277259265 & 1.26552017 & 0.987345249 \\
\hline
\end{tabular}

decreasing availability. A similar operation is performed in the process of network building. In actuality, a redundant channel with operational connections and the highest availability is used, if necessary.

\section{TRAFFIC RELIABILITY}

Let us consider the WAMS information channels for the power system, Fig. 2. The scheme of information connections with the distance scale is shown above in Fig. 3. Let us define the network conditions and characteristics. All data connections are made using fiber optics with a propagation delay $T_{s p}=5 \mathrm{~ns}$. Electronic delay is $T_{r e}=5 \mu \mathrm{s}$. The transmission speed is $v_{t r}=1 \mathrm{Mbit} / \mathrm{s}=$ $1048576 \mathrm{bit} / \mathrm{s}$ [17]. Measurement transmission frequency is $10 \mathrm{~Hz}$ or $T_{m s r}=0.1 \mathrm{~s}$. Control center is located at node 4 of the power system (Fig. 4), information routes of each information transmission channel under normal and emergency conditions are shown in Table 4, and its last column shows the connection of the source node to node 4 via bypass routes in the case of failure of the main route component. Note that failure of link 10-2 results in a complete loss of communication with node 10 . The initial data for the calculations are summarized in Table 10. Here, $b_{i n}$ and $b_{f r}$ in the third and fourth columns are the values of bytes associated directly with the corresponding link of the line; $\Sigma b^{n r}$ and $\Sigma b^{e m}$ are byte groups, including intermediate communication packets under both normal and emergency conditions caused by a failure of one of the links. $N$ is determined by the maximum frame under normal operating conditions and equals 5 .
The simulation results are shown in Tables 11 and 12, which indicate that the probability of information loss in the case of the calculated loads is very low. Let us consider the relationship between the information loss probability $q$ and the load intensity $\rho$ using the example of connection 7-4 with the rest of the same conditions. Using the same example, consider the effect of the number of storage sections $N$, Table 13 .

It is clear that for $N=0$, the probability of losing information is 1 since there is simply nowhere to receive it. With an increase in $N$, the value of q drops rather steeply, turning almost to zero already at $N=10$. It is also obvious that the greater the load intensity $\rho$ the greater the probability of information loss $q$. The rise is quick, which requires an increase in the number $N$ of receiver storage sections.

\section{THE COMPONENT CONTRIBUTION TO THE TOTAL RELIABILITY OF LOCAL INFORMATION NETWORK}

In the above sections, we determined the components of the WAMS information network reliability. In this section, we seek to determine the total reliability and the contribution of each component to this value. Initially, the question arose, what should be the contribution model of the component reliability should have? Let $R_{i, 1}$ be hardware reliability of the main $i$-th route, $R_{i, 2}$ be hardware reliability of the backup $i$-th route, $R_{i, 3}$ be the traffic reliability of the main $i$-th route, $R_{i, 4}$ be the traffic reliability of the backup $i$-th route. The contribution model can have one of the following schemes (Fig. 5). In scheme (a), the hardware and the traffic component are combined first, and then 
the main and backup routes are combined. In scheme (b), firstly, the hardware components of the main and backup routes are combined, then the traffic components of the same routes are combined, after which the obtained equivalents are connected sequentially. Equivalent values are determined as

$$
R_{a}=1-\left(1-R_{1} R_{3}\right)\left(1-R_{2} R_{4}\right)
$$$$
R_{b}=\left[1-\left(1-R_{1}\right)\left(1-R_{3}\right)\right]\left[1-\left(1-R_{2}\right)\left(1-R_{4}\right)\right],(13)
$$

With a large difference in the values of $R_{i}$, the relative difference between the equivalent values $Q_{i}=1-R_{i}$ can reach ten percent. Thus, for example, if the difference between $R_{i}$ is 0.1 , this difference lies between 39 and 49 percent (Table 14).

Let us consider such a relationship (Table 15) between the availability values of the route of fiber-optic information exchange channel ( $A_{\text {main }}$ and $A_{\text {redundant }}$ from Table 6) and the probabilities of the absence of information loss over a route due to the traffic load $\left(Q_{n r}\right.$ and $Q_{e m}$ from Table 12). Table 15 indicates that for the test scheme such a difference lies in the range of 0.01 to 0.85 percent, i.e., less than 1 percent. Therefore, when evaluating the components, any of the considered equivalent circuits can be used.

Further, we will estimate the contribution of reliability components to the local information network operation. It is more convenient to do that based on the component unavailability, i.e.,

$$
\begin{gathered}
\operatorname{Con}_{A, i}=\frac{1-A_{\text {channel with redun.,i }}}{\operatorname{Con}_{\Sigma, i}} 100 \% ; \\
\operatorname{Con}_{Q, i}=\frac{Q_{\Sigma w, i}^{a}}{\operatorname{Con}_{\Sigma, i}} 100 \% ; \operatorname{Con}_{S W, i}=\frac{1}{t_{S W} \cdot n \cdot \operatorname{Con}_{\Sigma, \mathrm{i}}} 100 \% ; \\
\operatorname{Con}_{\Sigma, i}=\left(\operatorname{Con}_{A, i}+\operatorname{Con}_{Q, i}+\operatorname{Con}_{S W, i}\right) \cdot 100 \%,
\end{gathered}
$$

where $\operatorname{Con}_{A, i}$ is a share of technical unavailability, $\operatorname{Con}_{Q, i}$ is a share of unavailability due to traffic. Here $Q_{\Sigma u, i}^{a}$ is used for an adverse event. $\mathrm{Con}_{S W, i}$ is a share of unavailability for

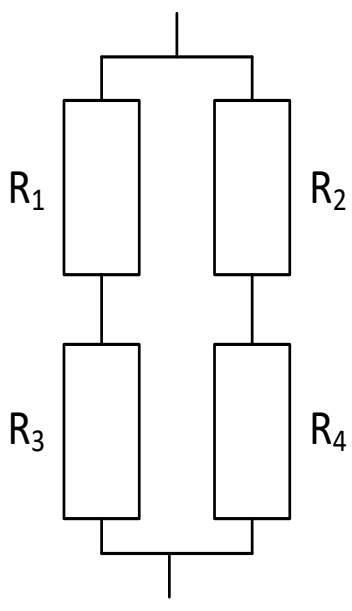

a)

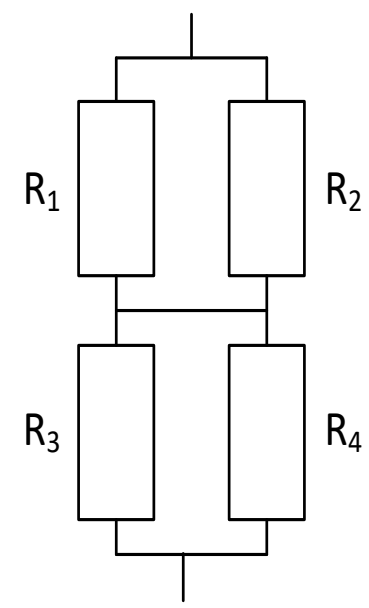

b)
Fig. 5. Models of the route reliability component contribution to the total reliability. software, $n$ is the number of the information source nodes. Here we rely on the assumption that the software is divided equally among the node devices, i.e., in our case $n=10$. $\mathrm{Con}_{\Sigma, i}$ is the sum of the shares of component unavailabilities. The total availability of a LIN route can be determined as

$$
A_{\Sigma, i}=A_{\text {channel with redun }, i} \cdot\left(1-Q_{\Sigma_{n, i}}^{a}\right) \cdot\left(1-\frac{1}{t_{S W} \cdot n}\right)
$$

For the considered scheme, the initial data on the fiberoptic network are summarized in Table 16. The calculation results are shown in Table 17.

Table 17 shows that the farther the source node from the dispatching node, the greater the weight of the hardware reliability component. On the other hand, the closer the source to the dispatching node, the heavier the traffic, which levels the total route availability out.

\section{CONCLUSIONS}

The proper functioning of the WAMS information network is ensured by four components of its reliability. These are hardware or technical reliability associated with the failure of transmission channel elements or destruction of the integrity of information transmission lines; software reliability related to errors in the development of exchange execution programs; traffic reliability determined by the time loss or distortion of data without a failure of the transmission channel elements; and resistance to external deliberate impact on the transmitted information. The influence of the latter component is discussed in many works, for example, $[12,13]$, which is why it is not considered in the paper.

Convenient algorithmization of the assessment of the components of the local information network reliability simplifies the implementation of computer applications of the assessment.

The reliability of hardware (PMUs, PDCs) of such a network largely depends on the reliability of information carriers (optical fiber, radio waves, and others) and devices that ensure its operation. The paper deals with an approach designed to determine the parameters of such reliability on the example of a 10-node power system. Thus, with the appropriate redundancy, the hardware availability of the network, including information sources (PMUs), exceeds three nines after the decimal point for fiber optics and is slightly less when information exchange occurs over power lines. The ways of increasing the hardware reliability of the information network are considered.

The traffic reliability component is determined by the load intensity of each link and the information receiving capabilities associated with the receiver storage capacity. It is worth noting that there is a strong dependence of the probability of information loss on the number of sections in the receiver storage device, whose increase makes it possible to compensate within some range for the growth of this probability with an increase in the load intensity. The test network traffic availability also exceeded three nines after the decimal point. 
In terms of software, the impact of the number of code lines on the value of this parameter is noted and its assessment is shown depending on the number of commands. A significant property of this index is its improvement with an increase in operating time. However, it can be incorrect due to new errors appearing when correction is made under operation. For the example of a WAMS program of 10 million code lines, the mean time between failures should be 285 years.

The study has revealed that despite different results obtained for various equivalent circuits, the error in their calculations for the range of requirements for their values lies within acceptable limits.

The contribution of the considered components to the total reliability has been assessed for the test scheme. The findings have shown that the greater the distance between the source node and the dispatching node, the greater the weight of the hardware reliability component. On the other hand, the closer the source to the dispatch center, the heavier the traffic, which equalizes the total availability of different routes.

\section{REFERENCES}

[1] A. G. Phadke and J. S. Thorp, "Synchronized Phasor Measurements and Their Applications," New York, NY, USA: Springer, 2008, $260 \mathrm{p}$.

[2] M. I. Uspensky, "Estimation of the functioning reliability for the device of synchronized vector measurements," Relay protection and automation, no. 3, pp. 39-44, 2017. (In Russian).

[3] Real-Time Application of Synchrophasors for Improving Reliability, NERC, Princeton, NJ, USA, 10/18/2010, 77 p. Available at: http://www.naspi.org (accessed 09.02.2020)

[4] V. N. Tsukanov, M. Ya. Yakovlev, "Fiber-optic technology. Practical Guide," Moscow, M., Russia: Infra-Engineering, 2014, 304 p. (In Russian).

[5] A. E. Davydov, P. I. Smirnov, A. I. Paramonov, "Design of telecommunication systems and networks. Calculation of communication network parameters and traffic analysis," St. Petersburg, Russia: ITMO University, 2016, 47 p. (In Russian).

[6] M. O. Asanov, V. A. Baransky, V. V. Rasin, "Discrete mathematics: graphs, matroids, algorithms," Moscow, M., Russia: RHD, 2001, 288 p. (In Russian).

[7] N. B. Zeliger, O. S. Chugreev, G. G. Yanovsky, "Designing networks and transmission systems for discrete messages," Moscow, M., Russia: Radio and communications, 1984, 177 p. (In Russian)

[8] Yu. M. Morozov, "Reliability of hardware and software systems," St. Petersburg, Russia: State Polytechnic University, 2011, 136 p. (In Russian)

[9] V. N. Shklyar, "Reliability of control systems," Tomsk, Russia: Publishing house of Tomsk Polytechnic University, 2009, 126 p. (In Russian)

[10] V. O. Chukanov, V. V. Gurov, E.V. Prokopyeva, "Methods of ensuring software and hardware reliability for computing systems," Russia, Presentation of the report at the seminar, .1-44 p. Available at: http://www.mcst.ru/files/5357 ec/ dd0cd8/50af39/000000/seminar_metody_obespecheniya _apparatno-programmnoy_nadezhnosti_vychislitelnyh_ sistem.pdf(In Russian). (accessed 12.03.2019)

[11] S. M. Borovikov, S. S. Dik, N. K. Fomenko, "A method for predicting applied software tools at the early stages of their development," Reports of the Belarusian State University of Informatics and Radioelectronics. 2019, no.5, pp. 45-51.

[12] Y. Zhang et al., "Cyber-Physical Security Analytics for Transactive Energy Systems," IEEE Trans. on Smart Grid, vol. 11, no. 2, pp. 931-941, March 2020.

[13] E. Martel, R. Kariger, P.-A. Graf, "Cyber Resilience in the Electricity Ecosystem: Principles and Guidance for Boards," Geneva, Switzerland, Centre for Cybersecurity and Electricity Industry Community, January 2019, 29 p. Available at: www3.weforum.org. (accessed 19.03.2020)

[14] M. I. Uspensky, S. O. Smirnov, "The Occurrence Reasons and Countermeasures to Power System Blackouts," The International Journal of Energy Engineering, no. 1, pp.1-8, 2014.

[15] W. Li, "Risk Assessment of Power Systems: Models, Methods, and Applications," New York, NY, USA: WileyIEEE Press, 2005, 325 p.

[16] "Reliability evaluation of the designed FOCL," Available at: http://www.icete.ru/pegibs-567-1.html. (In Russian). (accessed 23.11.2020)

[17] V. N. Gordienko et al., "Optical telecommunication systems. Textbook for high schools," Moscow, M., Russia: Hotline - Telecom, 2011, 368 p. (In Russian)

[18] M. I. Uspensky, I. V. Kyzrodev, "Power supply restoration in distributive networks," Reliability: Theory \& Applications, vol.2, no. 2, June, pp. 72-84. 2011.

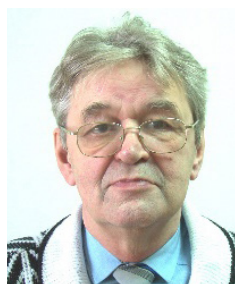

Michael Igorevich Uspensky graduated from Leningrad (St. Petersburg) Polytechnic Institute in 1971. He received his $\mathrm{Ph} . \mathrm{D}$. degree in engineering from Leningrad Polytechnic Institute in 1985. At present, he is a leading scientist at the Laboratory of Power Systems. M.I. Uspensky works for the Institute for Social, Economic and Energy Problems of the North at the Komi Science Center of the Ural Branch of the Russian Academy of Sciences in Syktyvkar, Russia.

His research interests include operating control and reliability of power system facilities. 